\title{
Comprehensive evaluation of the clinical utility of plasma EGFR test in non-small cell lung cancer patients with acquired resistance to first-line EGFR inhibitors
}

\author{
Hongsik Kim, Hyun Ae Jung, Se-Hoon Lee, Jin Seok Ahn, Myung-Ju Ahn, Keunchil Park, Jong-Mu Sun \\ Division of Hematology-Oncology, Department of Medicine, Samsung Medical Center, Sungkyunkwan University School of Medicine, Seoul, \\ Republic of Korea \\ Contributions: (I) Conception and design: JM Sun, H Kim; (II) Administrative support: H Kim; (III) Provision of study materials or patients: All \\ authors; (IV) Collection and assembly of data: H Kim; (V) Data analysis and interpretation: JM Sun, H Kim; (VI) Manuscript writing: All authors; (VII) \\ Final approval of manuscript: All authors. \\ Correspondence to: Jong-Mu Sun, MD, PhD. Division of Hematology-Oncology, Department of Medicine, Samsung Medical Center, Sungkyunkwan \\ University School of Medicine, 81 Irwon-ro, Gangnam-gu, Seoul 06351, Republic of Korea. Email: jongmu.sun@skku.edu.
}

\begin{abstract}
Background: Plasma epidermal growth factor receptor (EGFR) mutation tests are widely used when non-small cell lung cancer (NSCLC) patients acquire resistance to EGFR inhibitors. We comprehensively evaluated the clinical utility of plasma EGFR test.

Methods: We screened NSCLC patients who had a plasma EGFR test upon acquiring resistance to firstor second-generation EGFR inhibitors. Plasma EGFR tests were performed with the EGFR mutation test.

Results: A total of 355 patients were tested for plasma EGFR mutations, and T790M was detected in 83 patients (23\%). Of 79 patients who were tested multiple times, T790M was newly detected in 13 subsequent plasma tests. When initial plasma tests did not detect any EGFR mutation types, the detection rate of T790M in subsequent tests was very low $(9 \%, 5 / 56)$, while detection rates of T790M in subsequent tests increased $(35 \%, 8 / 23)$ in those individuals in whom sensitizing mutations had been detected in the initial plasma test $(\mathrm{P}=0.005)$. Paired plasma and tissue EGFR test results were available for 235 patients. Sensitivity and specificity of the plasma tests for T790M were 14\% and 87\%, respectively. Among 235 patients, 140 patients had tissue EGFR tests performed after T790M-negative plasma results were reported. The subsequent tissue test detected T790M in 61\% (44/72) of these patients when any EGFR mutations were not detected in prior plasma tests, while the detection rate of T790M in subsequent tissue tests was 37\% $(25 / 68)$ when sensitizing mutations were detected in prior plasma tests $(\mathrm{P}=0.004)$.

Conclusions: Because the sensitivity of plasma EGFR test for T790M is low, follow-up tissue or plasma tests are necessary. Presence or absence of a sensitizing mutation in the initial plasma tests can be used to determine which samples (tissue or plasma) should be submitted for further testing.
\end{abstract}

Keywords: Non-small cell lung cancer (NSCLC); epidermal growth factor receptor (EGFR); circulating tumor DNA (ctDNA); repeat tests; acquired resistance

Submitted Oct 19, 2020. Accepted for publication Jan 05, 2021.

doi: $10.21037 /$ tlcr-20-1128

View this article at: http://dx.doi.org/10.21037/tlcr-20-1128

\section{Introduction}

Epidermal growth factor receptor (EGFR) tyrosine kinase inhibitors (TKIs), including gefitinib, erlotinib and afatinib, are first-line therapies for EGFR-mutant advanced non- small cell lung cancer (NSCLC) patients $(1,2)$. However, most patients who are treated with first-line EGFR-TKIs acquire resistance after a median of 9.7 to 13 months $(3,4)$. Various resistant mechanisms towards first-line EGFR- 
TKIs are known; the most common resistant mechanism is the EGFR T790M mutation, which has been estimated to occur in approximately $50 \%$ to $60 \%$ of patients $(5,6)$.

Osimertinib, a third-generation TKI, showed good clinical outcomes in patients with EGFR T790M-positive tumors among patients who acquired resistance to prior EGFR TKIs $(7,8)$. Though the FLAURA trial showed superior overall survival (OS) of osimertinib as a first-line therapy in patients with sensitizing EGFR mutations (exon 19 deletion or L858R) compared with gefitinib for EGFR-mutant NSCLC (9), osimertinib is still used as a subsequent regimen after first- or second-generation EGFR TKIs in many countries, including Korea, due to its high cost. Therefore, when patients require subsequent treatment after treatment with first- or second-generation TKIs, it is important to evaluate the mechanism of resistance to prior EGFR TKIs.

A repeat tissue biopsy is the standard method for the detection of the EGFR T790M mutation (2). However, tissue biopsies are invasive and there is a high risk of complications (10). Tumors can release DNA in the blood (plasma), which is referred to as circulating tumor DNA (ctDNA), and this tumor DNA can easily be accessed through blood sampling.

The accuracy of plasma EGFR tests was evaluated in the AURA3 trial. The sensitivity of plasma EGFR test was $51 \%$ for the detection of T790M using tissue samples as a reference in patients who had acquired resistance to prior EGFR TKIs such as gefitinib, erlotinib, or afatinib (11). The College of American Pathologists (CAP) cautiously recommends at the level of 'Expert consensus opinion' that physicians may first select plasma EGFR tests to detect T790M in all patients who have acquired resistance to firstor second-generation EGFR TKIs, while reserving testing of tissue samples for patients with T790M-negative plasma (12). National Comprehensive Cancer Network (NCCN) adopted this recommendation, favoring the use of a plasma EGFR test as a screening method to detect T790M, irrespective of the feasibility of repeat tissue biopsy (2). The United States Federal Drug Administration (U.S. FDA) has not, however, come out in support of routine plasma testing for T790M, recommending the plasma EGFR test only for patients whose tumor is ineligible for repeat tissue biopsy.

The cobas ${ }^{\circledR}$ EGFR Mutation Test v2 (Roche Molecular Systems, Inc., Pleasanton, CA) has been developed to detect many EGFR mutation types, including T790M, as well as sensitizing mutations in both tissue and plasma samples. This method is commonly used as a companion diagnostic test to select patients who would likely benefit from
EGFR TKI treatment (13). Several years have passed since approval of the cobas ${ }^{\circledR}$ plasma EGFR test for the detection of T790M, and this test is widely used in clinical practice. However, the pattern of clinical implementation and the clinical utility of the plasma EGFR test for the detection of T790M have not been fully evaluated. Furthermore, the recommendations of the NCCN and US FDA are different regarding the sequence of implementation of plasma or tissue EGFR tests for the detection of T790M. Therefore, we evaluated the clinical utility of the plasma EGFR test, and provide suggestions for optimal incorporation of the plasma EGFR test in clinical practice.

The authors present the following article in accordance with the STARD reporting checklist (available at http:// dx.doi.org/10.21037/tlcr-20-1128).

\section{Methods}

This study was conducted in accordance with the Declaration of Helsinki (as revised in 2013). This study was approved by Institutional Review Board (IRB No. 2020-09-138) at Samsung Medical Center and individual consent for this retrospective analysis was waived.

\section{Patients}

We initially screened patients with advanced NSCLC who had acquired resistance after treatment with first- or second-generation EGFR-TKIs as the first-line therapy at Samsung Medical Center, Korea. Among them, we selected those patients whose samples had been analyzed with the plasma cobas ${ }^{\circledR}$ EGFR mutation test (cobas ${ }^{\circledR}$ EGFR mutation test v2, Roche Molecular Systems, Inc., Pleasanton, CA) at the time of acquired resistance. Patients who had also undergone a repeat tissue biopsy that was analyzed using tissue EGFR mutation test were further selected and analyzed.

\section{Sample collection and EGFR mutation test}

We performed tissue and blood (plasma) cell-free EGFR mutation tests for those patients who had experienced disease progression. The repeat tissue biopsy sample was formalin-fixed and paraffin-embedded. DNA was extracted and then evaluated to detect EGFR mutations. Serially collected blood samples had EGFR mutation tests performed using the cobas $^{\circledR}$ cell-free EGFR Mutation Test v2. Ten milliliters of blood was collected in a Cell- 
Free DNA collection tube (Roche). The subsequent process followed the protocol provided by the manufacturer of the cobas ${ }^{\circledR}$ EGFR Mutation Test v2.

\section{Statistical analyses}

The cut-off date for data collection was May 21, 2020. Descriptive statistics were used to summarize patient and tumor characteristics, EGFR mutation results, and treatment history. Associations between the results of the EGFR mutation test and clinical features were assessed using Fisher's exact test and the chi-square test. The agreement in sensitivity and specificity between the tissue and plasma EGFR mutation test results was assessed using the ClopperPearson exact method for binomial proportions. Clinical efficacy outcomes such as objective response rate (ORR), progression-free survival (PFS), and OS were evaluated on the basis of Response Evaluation Criteria in Solid Tumors (RECIST) version 1.1 by the investigators. PFS and OS analyses were performed on subgroup populations based on the plasma and tissue EGFR baseline mutation types and presence of the T790M mutation. PFS and OS were assessed by the Kaplan-Meier method and p-values from a log-rank test. IBM SPSS Statistics 25 (Armonk, NY, USA) was used for statistical analysis.

\section{Results}

From May 2018 to May 2020, 355 consecutive patients with available plasma EGFR tests acquired resistance to first-line EGFR-TKIs (plasma EGFR cohort). Among them, 235 patients had a repeat tissue biopsy and EGFR mutation test performed at the time of disease progression after treatment with first-line EGFR TKIs (paired plasma and tissue EGFR cohort) (Figure 1). Baseline characteristics of both the plasma EGFR cohort and the paired plasma and tissue EGFR cohort at the time of acquired resistance to the prior EGFR TKI are described in Table 1. Most patients had sensitizing EGFR mutations (exon 19 deletion or L858R) before starting first-line EGFR TKIs, such as gefitinib, erlotinib, afatinib, or dacomitinib. In the plasma EGFR cohort, the median number of metastatic sites was two, and $68 \%$ of patients had an extrathoracic metastasis.

\section{T790M mutation rates based on the plasma EGFR mutation test}

Median turnaround time of the plasma EGFR test was 5 days (interquartile range, IQR: 4-7). Of the total of 355 patients in the plasma EGFR cohort, 81 (23\%) were found to have the T790M mutation. Eighty-three patients (23\%) underwent multiple plasma EGFR test for the detection of T790M: two tests $(n=72)$, three tests $(n=10)$, and four tests $(\mathrm{n}=1)$ with a median time interval of 4.2 months (IQR: 2.5 to 8.6). Among 83 patients with multiple plasma tests, four were tested to confirm the first plasma T790M-positive result, and the other 79 patients were re-examined when prior plasma tests were T790M-negative. Among them, the T790M mutation was detected in $13(17 \%, 13 / 79)$ patients; 11 patients in the second test $(16 \%, 11 / 68)$ and two in the third test $(20 \%, 2 / 10)$.

We reviewed the initial plasma EGFR results in 13 and 66 patients with and without positive conversion to T790M, respectively, in subsequent plasma EGFR tests. Analysis of the first 13 plasma samples in the group positive for T790M conversion revealed the absence of any EGFR mutation types (EGFR wildtype) in 38\% (5/13) of patients, while $62 \%(8 / 13)$ had sensitizing EGFR mutations. Analysis of the first 66 plasma samples in the group without positive conversion to $\mathrm{T} 790 \mathrm{M}$ revealed that $77 \%(51 / 66)$ had EGFR wild type and 23\% (15/66) had baseline sensitizing EGFR mutations in the initial plasma tests. Sensitizing mutations were more commonly detected in the first plasma EGFR test in the T790M-positive conversion group than in the consistently T790M-negative group (62\% vs. $23 \%$, $\mathrm{P}=0.005$; Table 2).

\section{Concordance between tissue and plasma EGFR mutation test results}

Median turnaround time for the tissue EGFR test was 12 days (IQR: 9 to 14). In the paired plasma and tissue EGFR cohort ( $\mathrm{n}=235), 102(43 \%)$ patients had the T790M mutation in the tissue EGFR test and 32 (14\%) had the T790M mutation in the plasma EGFR test. In combined analyses of both plasma and tissue EGFR tests for the 235 patients, 14 patients (6\%) had both plasma- and tissueT790M-positive results, 88 (37\%) had plasma-T790Mnegative and tissue-T790M-positive results, 18 (8\%) had plasma-T790M-positive and tissue-T790M-negative results, and 115 (49\%) had both plasma- and tissue-T790Mnegative results (Figure 1).

When the tissue-based EGFR mutation results were used as the reference, the sensitivity and specificity of the plasma EGFR test for the T790M mutation was $14 \%$ (95\% CI, $9.5 \%$ to $18.7 \%$ ) and $87 \%$ (95\% CI, $81.3 \%$ to 


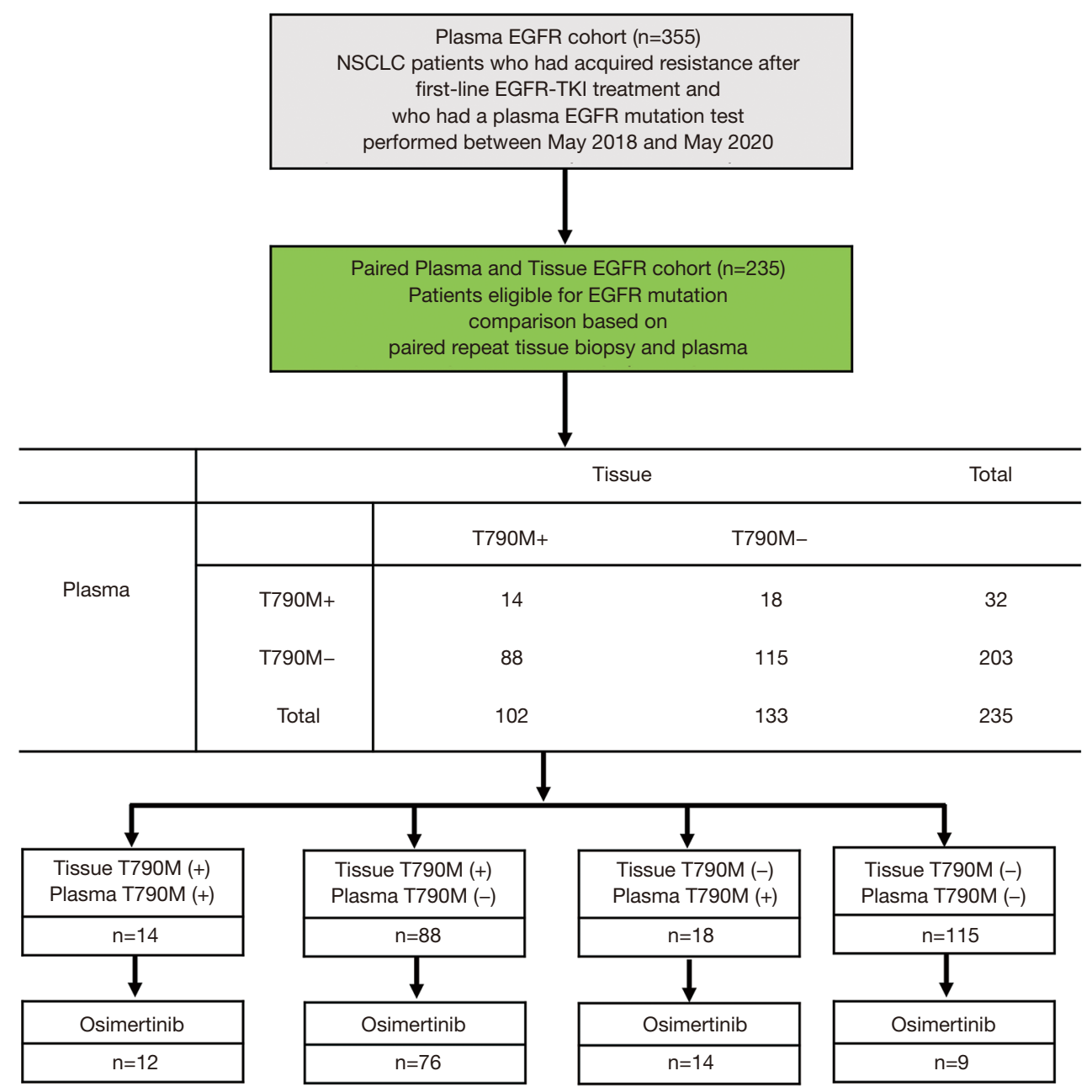

Figure 1 Flow diagram of the study population. EGFR, epidermal growth factor receptor; TKI, tyrosine kinase inhibitor.

90.5\%), respectively (Figure 1).

\section{Timing of plasma and tissue EGFR tests in the paired EGFR cohort}

We analyzed temporal relationship between the timing of plasma or tissue biopsies. The first group comprised patients in whom sequential plasma EGFR tests were performed followed by tissue EGFR tests: $140(60 \%)$ patients had a tissue biopsy performed after receiving a plasma T790Mnegative result. Median time interval between the acquisition of plasma and tissue specimens was 2.0 months (IQR, 0.7 to 3.3 months). Among them, the T790M mutation was detected in $69(49 \%)$ patients in subsequent tissue EGFR tests. Of these patients, 64\% (44/69) were EGFR wildtype while $36 \%(25 / 69)$ had a sensitizing EGFR mutation in the initial plasma EGFR test. In the other 71 patients without T790M-positive conversion on subsequent tissue specimens, 39\% (28/71) were EGFR wild type and $61 \%(43 / 71)$ had sensitizing mutations in prior plasma EGFR tests. There were more patients who had an EGFR wild-type result in prior plasma tests in the T790Mpositive conversion group than in the consistently T790Mnegative group ( $64 \%$ vs. $39 \%, \mathrm{P}=0.004$; Table 3).

The second group comprised those patients in whom tissue EGFR tests were followed by plasma EGFR tests: $36(15 \%)$ patients had a plasma test after a tissue T790Mnegative result, and the median interval between the acquisition of tissue and plasma samples was 7.2 months (IQR, 3.9 to 13.6 months). Among them, 11 (31\%) patients had a T790M mutation based on analysis of subsequently acquired plasma specimens. The prior tissue EGFR results 
Table 1 Patient characteristics

\begin{tabular}{|c|c|c|}
\hline Characteristics & Plasma EGFR cohort $(n=355)$ & Paired plasma and tissue EGFR cohort $(n=235)$ \\
\hline \multicolumn{3}{|l|}{ Sex, no. [\%] } \\
\hline Male & $137[39]$ & 92 [39] \\
\hline Female & $218[61]$ & $143[61]$ \\
\hline \multicolumn{3}{|l|}{ Smoking status, no. [\%] } \\
\hline Never & $241[68]$ & $160[68]$ \\
\hline Former or current & 114 [32] & 75 [32] \\
\hline \multicolumn{3}{|c|}{ Number of metastatic sites } \\
\hline Median, $\mathrm{N}(\min , \max )$ & $2(0,7)$ & $2(0,6)$ \\
\hline \multicolumn{3}{|c|}{ Extrathoracic metastasis, no. [\%] } \\
\hline No & $113[32]$ & 89 [38] \\
\hline \multicolumn{3}{|l|}{ Diameter of largest tumor } \\
\hline Median, mm (range) & $27(5-138)$ & $30(5-102)$ \\
\hline \multicolumn{3}{|l|}{ Prior TKI therapy, no. [\%] } \\
\hline Gefitinib & 139 [39] & $93[40]$ \\
\hline Erlotinib & 48 [14] & 32 [14] \\
\hline Afatinib & $167[47]$ & $109[46]$ \\
\hline Dacomitinib & $1[0]$ & $1[0]$ \\
\hline L861Q & $2[1]$ & $1[1]$ \\
\hline Others $^{\dagger}$ & $3[1]$ & $3[1]$ \\
\hline
\end{tabular}

${ }^{\dagger}$, Three cases were positive for both G719X and D19, S768I and L858R, exon 20 insertion and L858R based on tumor tissue assay at diagnosis. EGFR, epidermal growth factor receptor; TKI, tyrosine kinase inhibitor.

Table 2 T790M detection in Serial plasma EGFR tests $(\mathrm{n}=79, \mathrm{P}=0.005)$

\begin{tabular}{lcc}
\hline EGFR test results & $\begin{array}{c}\text { Sensitizing mutation(-) /T790M(-) } \\
\text { at initial plasma test }(n=56)\end{array}$ & $\begin{array}{c}\text { Sensitizing mutation(+) /T790M(-) } \\
\text { at initial plasma test }(n=23)\end{array}$ \\
\hline T790M $(+)$ conversion at subsequent Plasma tests $(n=13)$ & $5(9 \%)$ & $8(35 \%)$ \\
Consistent T790M(-) at subsequent plasma tests $(n=66)$ & $51(91 \%)$ & $15(65 \%)$ \\
\hline
\end{tabular}


Table 3 T790M detection in Plasma EGFR tests followed by tissue tests ( $\mathrm{n}=140, \mathrm{P}=0.004)$

\begin{tabular}{lcc}
\hline EGFR test results & $\begin{array}{c}\text { Sensitizing mutation(-)/T790M }(-) \\
\text { at initial plasma test }(n=72)\end{array}$ & $\begin{array}{c}\text { Sensitizing mutation(+)/T790M }(-) \\
\text { at initial plasma test }(\mathrm{n}=68)\end{array}$ \\
\hline T790M $(+)$ conversion at subsequent tissue tests $(n=69)$ & $44(61 \%)$ & $25(37 \%)$ \\
Consistent T790M $(-)$ at subsequent tissue tests $(n=71)$ & $28(39 \%)$ & $43(63 \%)$ \\
\hline
\end{tabular}

of these 11 patients with positive conversion to T790M in subsequent plasma tests were reviewed and we found that sensitizing EGFR mutations were present based on the prior tissue EGFR test in all cases (11/11, 100\%), similar to the detection rate of sensitizing EGFR mutations in prior plasma tests in the 25 patients with consistently T790Mnegative results $(21 / 25,84 \%, \mathrm{P}=0.29)$.

The third group comprised 59 patients with simultaneous plasma and tissue EGFR tests: seven (12\%) were T790Mpositive for only their plasma specimens, 19 (32\%) were T790M-positive for only their tissue samples, 14 (24\%) were T790M-positive for both their tissue and plasma samples, and 19 (32\%) were T790M-negative for both tissue and plasma samples.

In these three sequential test groups, the median diameter of the largest tumor was much larger in the simultaneous testing group $(33.5 \mathrm{~mm})$ or tissue-followedby-plasma EGFR testing group $(33.0 \mathrm{~mm})$ than the plasmafollowed-by-tissue testing group $(28.0 \mathrm{~mm})(\mathrm{P}=0.02)$. However, there was no difference in other indicators of tumor burden between the three groups, such as the number of metastatic sites $(\mathrm{P}=0.08)$ or extrathoracic metastasis $(\mathrm{P}=0.86)$.

\section{Clinical efficacy outcomes of osimertinib according to plasma or tissue T790M results}

Of 235 patients in the paired plasma and tissue EGFR cohort, 111 patients were treated with osimertinib. Twelve patients were treated with osimertinib in the plasmaand tissue-T790M-positive group, 76 in the tissue-onlyT790M-positive group, 14 in the plasma-only-T790Mpositive group, and nine in the plasma- and tissue-T790Mnegative group (Figure 1).

The ORR for osimertinib was $67 \%$ in patients with both plasma- and tissue-T790M positivity, 78\% in patients with only tissue-T790M-positivity, $57 \%$ in patients with only plasma-T790M-positivity, and $33 \%$ in patients with both tissue and plasma T790M-negativity $(\mathrm{P}=0.03)$. Irrespective of the plasma T790M results, the ORR for osimertinib in the tissue-T790M-positive group $(\mathrm{n}=88)$ was $76 \%(67 / 88)$, which is higher than that in the tissueT790M-negative group (48\%; 11/23) ( $\mathrm{P}=0.008)$. However, the ORR for osimertinib was $62 \%(16 / 26)$ in the plasmaT790M-positivity group $(n=26)$ irrespective of the tissue T790M results, which was numerically lower than the ORR of $73 \%(62 / 85)$ for the plasma-T790M-negative group $(\mathrm{n}=85 ; \mathrm{P}=0.27)$.

Median PFS for osimertinib was 8.6 months (95\% CI, 3.7 to 13.5 months) in both plasma- and tissueT790M positive patients, 13.0 months (95\% CI, 9.2 to 16.7 months) in the only tissue-T790M-positive group, 8.8 months (95\% CI, 6.7 to 10.9 months) in the only plasma-T790M-positive group, and 6.8 months (95\% CI, 3.9 to 9.8 months) in patients who were both plasma and tissue $\mathrm{T} 790 \mathrm{M}$ negative $(\mathrm{P}=0.002$, Figure $2 A)$. Irrespective of the plasma T790M results, the median PFS for osimertinib in the tissue-T790M-positive group was 13.0 months (95\% CI, 9.4 to 16.6 months), which is significantly longer than the 7.2 months (95\% CI, 6.1 to 8.3 months) for the tissue-T790M-negative group $(\mathrm{P}<0.001$; Figure $2 B)$. Median PFS for osimertinib, however, in the plasma-T790M-positive patients, irrespective of tissue-T790M result, was 8.8 months (95\% CI, 7.1 to 10.5 months), which was numerically shorter than the median PFS (12.3 months, 95\% CI, 8.9 to 15.7 months) in the plasma-T790M-negative group $(\mathrm{P}=0.10$, Figure $2 C)$.

\section{Shedding of EGFR mutant cells and its prognostic role}

Of 355 patients in the plasma EGFR cohort, sensitizing EGFR mutations were detected with $(\mathrm{n}=81)$ or without T790M ( $\mathrm{n}=147$ ) in 228 plasma samples (the shedder group), while no EGFR mutations were detected in 127 plasma samples (the non-shedder group). There were no clear associations between EGFR mutant ctDNA shedding status and age $(\mathrm{P}=0.28)$, sex $(\mathrm{P}=0.49)$, smoking status $(\mathrm{P}=0.51)$, or diameter of the largest tumor $(\mathrm{P}=0.61)$. However, ctDNA shedding was more frequently found in patients with two or 

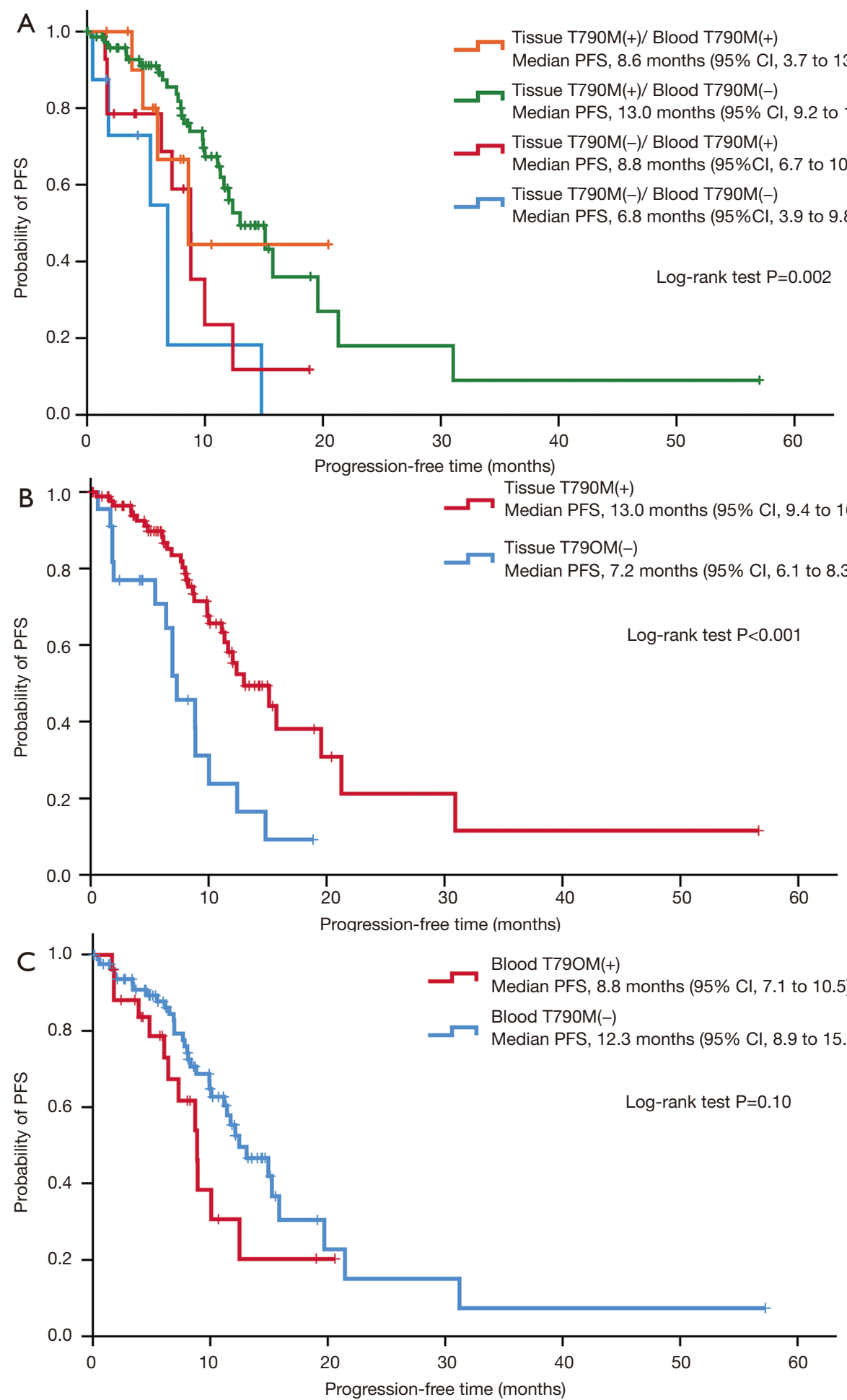

Figure 2 Kaplan-Meier curves of PFS based on T790M-positive (T790M+) and T790M-negative (T790M-) subpopulations treated with osimertinib. (A) Patients who were T790M+ on tissue or blood samples exhibited longer PFS than patients who were both T790M- (P=0.002). (B) Patients who were tissue T790M+ exhibited significantly longer PFS than patients who were tissue T790M- $(\mathrm{P}<0.001)$. (C) There was no difference in PFS based on blood T790M results $(\mathrm{P}=0.10)$. CI, confidence interval; PFS, progression-free survival. 
more metastatic sites $(\mathrm{P}=0.005)$ or extrathoracic metastatic lesions $(\mathrm{P}=0.012)$.

Median PFS for shedders who received first-line EGFR TKI treatment ( $\mathrm{n}=228)$ was 14.0 months (95\% CI, 12.4 to 15.6 months), which was significantly shorter than the median PFS (18.7 months, 95\% CI, 16.2 to 21.2 months) of non-shedders $(\mathrm{P}=0.006)$. Analysis of OS according to detection of sensitizing EGFR mutations in plasma tests was performed after excluding 185 patients treated with osimertinib to rule out the confounding effects of osimertinib therapy. Median OS, defined as the period from the start of first-line therapy to death, was 40.9 months (95\% CI, 33.1 to 48.8 months) for shedders $(n=110)$, which was significantly shorter than the median OS of non-shedders (46.7 months, 95\% CI, 41.5 to 51.9 months).

\section{Discussion}

The current study showed that the detection rate of T790M using plasma EGFR test in patients who acquired resistance to prior EGFR TKIs was $23 \%$. Using tissue EGFR results as a reference value, sensitivity was also low (14\%). These values are much lower than those reported by the AURA3 trial, where the sensitivity of plasma EGFR test for T790M was reported to be $51 \%$. However, our data are consistent with other real world data. One Canadian group reported that the T790M detection rate of droplet digital PCR was 24\% (82/343) (14), and digital droplet PCR is considered more sensitive than the cobas ${ }^{\circledR}$ test $(11)$.

The reason for the unexpectedly lower T790M detection rate in the plasma EGFR test in real world practice is not clear from our study, but it may in part be due to selection bias associated with the feasibility of tissue biopsy. The AURA3 trial enrolled patients on the basis of tissue T790M positive results, and additionally acquired paired plasma samples for exploratory evaluation of the accuracy of plasma EGFR tests. Therefore, all patients in AURA3 were eligible for repeat tissue biopsy. Among the paired plasma and tissue EGFR cohort in our study, however, about $60 \%$ of patients first had a plasma EGFR test. If our practice followed the FDA recommendation (plasma EGFR test for only ineligible for tissue repeat biopsy), tissue biopsy might be challenging in those patients, although tissue biopsy was successfully performed at last in this subgroup. These patients can be suspected to have a lower tumor burden and subsequently lower ctDNA volume than the detection limit of the plasma EGFR test. This hypothesis is in part supported by subgroup analysis of 59 patients who had a simultaneous plasma and tissue EGFR test results. Among them, the detection rate and sensitivity for T790M by the plasma EGFR test was 36\% (21/59) and 42\% (14/33), respectively, which were slightly higher than the values $(23 \%$ and $14 \%$, respectively) from our whole population. It is also supported that median diameter of the largest tumor was much shorter in the groups where plasma testing was done before the tissue EGFR test than the groups where tissue EGFR testing was tried before or simultaneously with the plasma tests.

Clinical advantages of plasma EGFR test plasma are its easy accessibility and the ability to perform repeat tests. Among 355 patients in the plasma EGFR cohort, 23\% had the plasma EGFR test performed multiple times, and about $17 \%$ of patients were newly found to have T790M. Additionally, among 36 patients with initial T790Mnegative tissue, 11 patients tested T790M-positive on subsequent plasma tests, which were performed after a relatively long-term interval (median 7.2 months). These results suggest temporal variation in the volume of T790Mpositive clones as well as tumoral heterogeneity. Therefore, subsequent plasma EGFR tests performed at long-term intervals would be beneficial in patients with initial T790Mnegative plasma or tissue results.

We also attempted to evaluate who would derive the most benefit from multiple or repeated plasma or tissue EGFR tests, and analyzed 79 patients who underwent multiple plasma tests with initial T790M-negative plasma results. Of 56 EGFR wildtype patients (no sensitizing mutation and T790M) based on the initial plasma test, only five cases (9\%) had the T790M mutation on subsequent plasma EGFR tests, while eight $(35 \%)$ of 23 patients with sensitizing mutations on the initial plasma test had the T790M mutation detected in subsequent plasma tests $(\mathrm{P}=0.005)$. This suggests that tumors that do not initially shed EGFRpositive clones remain non-shedders. However, of the 140 patients in whom a subsequent tissue biopsy was performed after an T790M-negative plasma result, 72 patients were EGFR wild-type and 68 had sensitizing mutations in the initial plasma tests. Of 72 patients with EGFR wild-type plasma, $61 \%$ were T790M-positive on the subsequent tissue test, while $37 \%$ of 68 patients with initial sensitizing mutation-positive plasma test results tested T790M positive $(\mathrm{P}=0.004)$. Based on our results, if the first plasma EGFR test result is negative for both sensitizing mutations and T790M, the possibility of detection of T790M is much higher on subsequent tissue biopsy rather than a repeated plasma test, with the detection rate of T790M increasing 




Figure 3 Optimal incorporation of plasma and tissue EGFR tests at acquired resistance to first- or second-generation EGFR TKIs. EGFR, epidermal growth factor receptor; TKI, tyrosine kinase inhibitor.

from $9 \%$ to $61 \%$ (Tables 2,3 ). If the initial plasma test is positive for sensitizing EGFR mutations, the detection rate of T790M is likely to be similar for subsequent plasma (35\%) and tissue (37\%) samples (Tables 2,3). Taken together, these results indicate that tissue biopsy should be performed after a test result of EGFR wild-type based on the initial plasma sample, while testing of either subsequent plasma or tissue samples is acceptable after detection of a sensitizing mutation in the initial plasma sample (Figure 3).

The efficacy of osimertinib in patients with discordant plasma and tissue T790M status was evaluated. ORR and PFS of patients who were plasma T790M-positive and tissue T790M-negative were inferior to those of patients who were tissue T790M-positive and plasma T790Mnegative (ORR: $57 \%$ vs. $78 \%$, median PFS: 8.8 vs. 13.0 months), which was compatible with the AURA3 trial (11). The reason for the lower efficacy of osimertinib in patients with T790M-positive plasma and T790M-negative tissue is not clear, but T790M clones may comprise only a small proportion of all resistant tumor clones, or other resistance mechanisms are likely to coexist in patients in whom T790M is detected in the plasma sample only.

The level of ctDNA is an important determinant of the sensitivity of the plasma EGFR mutation for detecting T790M as well as sensitizing mutations. The amount of ctDNA is closely associated with tumor burden (15). In our plasma EGFR cohort, no EGFR mutations were detected in the plasma samples of 127 patients. These non-shedders had a longer PFS and OS than those with EGFR mutationpositive plasma (shedders). These data indicate that the plasma EGFR test performed after acquisition of resistance to EGFR TKIs can be used a prognostic marker.

Our study illustrates the real-world pattern of clinical implementation of the plasma EGFR test. In the paired plasma and tissue cohort, about $60 \%$ of patients had their plasma tested first followed by a repeat tissue biopsy after a report of T790M-negative plasma, consistent with the NCCN recommendations (2). However, this proportion could be overestimated because some patients who underwent tissue biopsy first without subsequent plasma tests were excluded from our study population.

Our current study did not indicate which sequence of EGFR tests is better upon the acquisition of resistance; plasma first or tissue first. However, the detection rate of T790M was very low in the plasma EGFR test, and subsequent plasma or tissue EGFR tests should be tried at an appropriate time interval. Another unique finding of the current study is that tissue is better than plasma when performing a follow-up EGFR test to detect T790M if the initial plasma EGFR test shows wild-type (Figure 3). However, these findings and suggestions are drawn from a limited number of patients and should be further evaluated in larger patient populations.

\section{Conclusions}

Because the sensitivity of plasma EGFR test for T790M is low, follow-up tissue or plasma tests are necessary. Presence or absence of a sensitizing mutation in the initial plasma tests can be used to determine which samples (tissue or 
plasma) should be submitted for further testing.

\section{Acknowledgments}

Funding: This work was supported by the Bio \& Medical Technology Development Program of the National Research Foundation (NRF) funded by the Korean government (MSIT) (No. NRF-2017M3A9G5060259).

\section{Footnote}

Reporting Checklist: The authors have completed the STARD reporting checklist. Available at http://dx.doi.org/10.21037/ tlcr-20-1128

Data Sharing Statement: Available at http://dx.doi. org/10.21037/tlcr-20-1128

Conflicts of Interest: All authors have completed the ICMJE uniform disclosure form (available at http://dx.doi. org/10.21037/tlcr-20-1128). The authors have no conflicts of interest to declare.

Ethical Statement: The authors are accountable for all aspects of the work in ensuring that questions related to the accuracy or integrity of any part of the work are appropriately investigated and resolved. This study was conducted in accordance with the Declaration of Helsinki (as revised in 2013). This study was approved by Institutional Review Board (IRB No. 2020-09-138) at Samsung Medical Center and individual consent for this retrospective analysis was waived.

Open Access Statement: This is an Open Access article distributed in accordance with the Creative Commons Attribution-NonCommercial-NoDerivs 4.0 International License (CC BY-NC-ND 4.0), which permits the noncommercial replication and distribution of the article with the strict proviso that no changes or edits are made and the original work is properly cited (including links to both the formal publication through the relevant DOI and the license). See: https://creativecommons.org/licenses/by-nc-nd/4.0/.

\section{References}

1. Planchard D, Popat S, Kerr K, et al. Metastatic nonsmall cell lung cancer: ESMO Clinical Practice Guidelines for diagnosis, treatment and follow-up. Ann Oncol 2018;29:iv192-237. Erratum in: Ann Oncol 2019;30:863-70.

2. National Comprehensive Cancer Network. Non-

Small Cell Lung Cancer. Version 6. 2020. Accessed June 15, 2020. Available online: https://www.nccn.org/ professionals/physician_gls/pdf/nscl.pdf

3. Park K, Tan EH, O'Byrne K, et al. Afatinib versus gefitinib as first-line treatment of patients with EGFR mutationpositive non-small-cell lung cancer (LUX-Lung 7): a phase 2B, open-label, randomised controlled trial. Lancet Oncol 2016;17:577-89.

4. Rosell R, Carcereny E, Gervais R, et al. Erlotinib versus standard chemotherapy as first-line treatment for European patients with advanced EGFR mutation-positive non-small-cell lung cancer (EURTAC): a multicentre, open-label, randomised phase 3 trial. Lancet Oncol 2012;13:239-46.

5. Lee K, Kim Y, Jung HA, et al. Repeat biopsy procedures and T790M rates after afatinib, gefitinib, or erlotinib therapy in patients with lung cancer. Lung Cancer 2019;130:87-92.

6. Yu HA, Arcila ME, Rekhtman N, et al. Analysis of tumor specimens at the time of acquired resistance to EGFR-TKI therapy in 155 patients with EGFR-mutant lung cancers. Clin Cancer Res 2013;19:2240-7.

7. Janne PA, Yang JC, Kim DW, et al. AZD9291 in EGFR inhibitor-resistant non-small-cell lung cancer. $\mathrm{N}$ Engl J Med 2015;372:1689-99.

8. Mok TS, Wu YL, Ahn MJ, et al. Osimertinib or PlatinumPemetrexed in EGFR T790M-Positive Lung Cancer. N Engl J Med 2017;376:629-40.

9. Soria JC, Ohe Y, Vansteenkiste J, et al. Osimertinib in Untreated EGFR-Mutated Advanced Non-Small-Cell Lung Cancer. N Engl J Med 2018;378:113-25.

10. Overman MJ, Modak J, Kopetz S, et al. Use of research biopsies in clinical trials: are risks and benefits adequately discussed? J Clin Oncol 2013;31:17-22.

11. Papadimitrakopoulou VA, Han JY, Ahn MJ, et al. Epidermal growth factor receptor mutation analysis in tissue and plasma from the AURA3 trial: Osimertinib versus platinum-pemetrexed for T790M mutationpositive advanced non-small cell lung cancer. Cancer 2020;126:373-80.

12. Lindeman NI, Cagle PT, Aisner DL, et al. Updated Molecular Testing Guideline for the Selection of Lung Cancer Patients for Treatment With Targeted Tyrosine Kinase Inhibitors: Guideline From the College of American Pathologists, the International Association for the Study of Lung Cancer, and the Association for 
Molecular Pathology. J Thorac Oncol 2018;13:323-58.

13. United States Federal Drug Administration. List of Cleared or Approved Companion Diagnostic Devices (In Vitro and Imaging Tools). Accessed August 1, 2020. Available online: https:/www.fda.gov/medical-devices/ vitro-diagnostics/list-cleared-or-approved-companiondiagnostic-devices-vitro-and-imaging-tools

14. Spence T, Perera S, Weiss J, et al. Clinical implementation of circulating tumour DNA testing for EGFR T790M for detection of treatment resistance in non-small cell lung cancer. J Clin Pathol 2020. J Clin Pathol 2021;74:91-7.

15. Zhu YJ, Zhang HB, Liu YH, et al. Quantitative cell-free circulating EGFR mutation concentration is correlated with tumor burden in advanced NSCLC patients. Lung Cancer 2017;109:124-7.
Cite this article as: Kim H, Jung HA, Lee SH, Ahn JS, Ahn MJ, Park K, Sun JM. Comprehensive evaluation of the clinical utility of plasma EGFR test in non-small cell lung cancer patients with acquired resistance to first-line EGFR inhibitors. Transl Lung Cancer Res 2021;10(2):878-888. doi: 10.21037/tlcr20-1128 